\title{
Assessment of theories for free vibration analysis of homogeneous and multilayered plates
}

\author{
Erasmo Carrera \\ Dipartimento di Ingegneria Aerospaziale, Politecnico di Torino, Corso Duca degli Abruzzi 24, 10129, Torino, Italy \\ E-mail: erasmo.carrera@polito.it
}

Professor Bruno Piombo was one of Professor Ferrari's team-mate when Professor Ferrari was head of the 'Istituto di Aerodinamica e Meccanica Applicata' IAMA. About twenty five years ago, right after the new organization of the Italian University, some of the IAMA Scientists including Piombo joined the Department of Mechanics others went to the Aerospace Department. In a certain sense this work is aimed at being a tribute to the memory of Bruno Piomboof both the author and those former IAMA's Scientists who were colleagues and friends of Bruno and who now belong to the Aerospace Department.

\begin{abstract}
This paper assesses classical and advanced theories for free vibrational response of homogeneous and multilayered simply supported plates. Closed form solutions are given for thick and thin geometries. Single layer and multilayered plates made of metallic, composite and piezo-electric materials, are considered. Classical theories based on Kirchhoff and Reissner-Mindlin assumptions are compared with refined theories obtained by enhancing the order of the expansion of the displacement fields in the thickness direction $z$. The effect of the Zig-Zag form of the displacement distribution in $z$ as well as of the Interlaminar Continuity of transverse shear and normal stresses at the layer interface were evaluated. A number of conclusions have been drawn. These conclusions could be used as desk-bed in order to choose the most valuable theories for a given problem.
\end{abstract}

\section{Introduction}

Layered structures are increasingly used in aerospace, automotive and ship vehicles. The most common and best known examples of multilayered structures are sandwich panels. Many other layered materials have been introduced.

The so-called advanced composite materials were developed as part of aerospace vehicles during the second part of the last century. Nowadays there are examples of fighter and commercial aircraft, helicopters gliders and racing cars whose structures are made entirely of composite materials.

Other examples of layered structures are: thermal protected structures in which layers with high thermal properties are used as thermal skins; biomedical retina; advanced optical mirrors; semiconductor technologies; intelligent structures that embed piezo-layers and which are used as sensors and/or actuators to build a closed loop controlled 'smart' structure.

The analysis, design and construction of multilayered structures is a cumbersome subject. So many different, complicated and new problems arise to add to those that are already known for traditional homogeneous one layered, isotropic structures. See the review by Bogdanovich and Sierakowsky [2]. Of all the possible topics, this review covers those aspects that are related to two-dimensional modeling of layered structures with respect to their vibrational response.

The accurate evaluation of the vibrational response of layered plates plays a fundamental role in many applications in the field of sound, shock and vibration. However, two dimensional modelings of multilayered structures deserve 


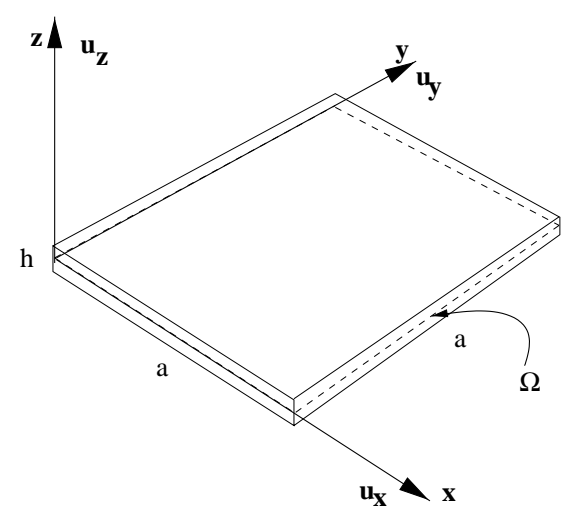

Fig. 1. Geometry and notations.

amendments to the classical theories that were originally developed for homogeneous plates. Among the necessary improvements mention can be made of the fulfillment of continuity of displacements Zig-Zag effects (ZZ) and of transverse shear and normal stresses Interlaminar Continuity (IC) at the interface between two adjacent layers. First Lekhnitskii [10], Ambartsumian [1] and than Reissner [1] proposed theories including ZZ and IC (see the historical review on Zig-Zag theories that has recently been made by Carrera [7]). Review articles on the papers, that are available on these subjects, can be found in Grigoliuk and Kulikov [8], Reddy and Robbins [16], Vasiliev and Lur'e [18], Lur'e and Shumova [11], Carrera [4-6] and Qatu [13,14].

Of all the refined theories, a convenient distinction can be made between models in which the number of the unknown variables is independent of or dependent on the number of constitutive layers of the plate. According to Reddy [15], we can assign the Equivalent Single Layer Models (ESLMs) to the first group while Layer Wise Models (LWMs) is used to denote the others. LWMs are computationally expensive and the use of ESLMs is preferred in most practical applications.

Recent works by the authors [4,7] have proposed a unified formulation for classical and advanced theories for layered structures. These are all based on both the Principle of Virtual Displacements (PVD) and the Reissner Mixed Variational Theorem (RMVT) [17]. As a key-point of this formulation, the governing equations are written in terms of a few fundamental nuclei, whose form is independent of the used set of base functions that are employed to express the unknown variables in the thickness direction. In this paper, this unified formulation is applied to assess the free vibration response of multilayered, simply supported plates. Closed form solutions are given for various plate problems. Results related to classical theories are compared to advanced ones. The effect of $\mathrm{ZZ}$ and IC, variable description (ESLM vs LWM) as well as of the the order of the expansion of the unknown variables in the thickness direction were investigated for all the considered problems. The paper has been organized as follows. Section 2 describes the compared theories while Section 3. deals with the numerical investigation that was carried out. Details of the governing equations as well as of the solution procedure have been omitted. These can all be found in the already mentioned author's works $[4,7]$.

\section{Plate theories compared in this work}

Plate theories can be developed according to various techniques and methodologies. A complete discussion on available technique has been provided in the already mentioned review articles. Herein the attention has been focused on the so called axiomatic approaches. In this framework the following cases are considered.

1. Classical theories based on well known Kirchhoff and Reissner-Mindlin type assumptions, namely CLT and FSDT.

2. Enhancement of FSDT to include transverse normal strains.

3. Refinements of FSDT by adding higher order terms in the displacement model.

4. Description of ZZ effect for the refined theories at point 2, by introducing the Murakami Zig-Zag functions. 


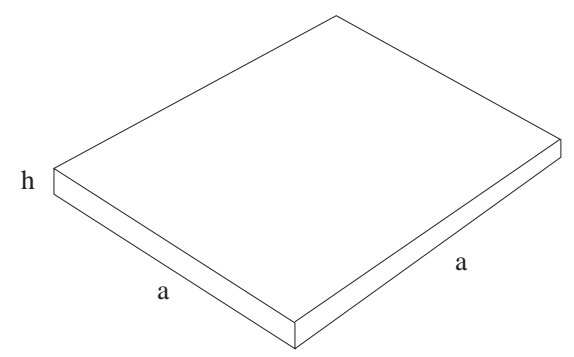

Fig. 2. Isotropic plates.

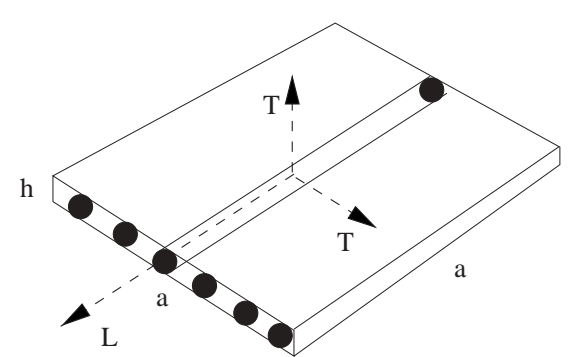

Fig. 3. Composite plate made of an orthotropic layer.

5. Mixed form of theories at point 2 to include IC.

6. Mixed form of theories at point 3 to include both $\mathrm{ZZ}$ and IC.

7. Layer-wise form of theories at point 2.

8. Layer-wise mixed form of theories at point 2 to include.

These theories are briefly described in the subsequent paragraphs.

CLT, Classical lamination Theory. The geometry and notations of the considered plate are given Fig. 1.

The simplest known plate theory is based on Kirchhoff thin plate assumptions, namely Classical Lamination Theory (CLT) (see [15]). Transverse shear strains as well as transverse normal strains are neglected with respect to in-plane ones. In the framework of CLT applications, resulting displacement model can be written:

$$
\begin{aligned}
u_{i} & =u_{i}^{0}-z u_{z,{ }_{i}} i=x, y \\
u_{z} & =u_{z}^{0}
\end{aligned}
$$

where superscript ${ }^{0}$ denotes values of displacement on $\Omega$. Comma denotes partial derivatives.

FSDT, First order Shear Deformation Theory. The inclusion of transverse shear strains leads to the following form of previous equations.

$$
\begin{aligned}
& u_{i}=u_{i}^{0}+z \phi_{i} i=x, y \\
& u_{z}=u_{z}^{0}
\end{aligned}
$$

This model is known as Reissner-Mindlin plate theory, namely First order Shear Deformation Theory, FSDT (see [15]). It consist in a first order Taylor-type expansion of displacement unknown in the neighborhood of the reference surface $\Omega$. $\phi_{x}, \phi_{y}$ takes therefore the meaning of rotations of the normal to $\Omega$ in the two planes $x-z$ and $y-z$, respectively.

FSDT model is herein re-written according to the following notations:

$$
u(x, y, z)=F_{0}(z) u_{0}(x, y)+F_{1}(z) u_{1}(x, y)
$$

The polynomials used in the expansion take the following values, 
Table 1

One layered isotropic plate made of Aluminum Alloys. Circular frequency parameter $\omega \times \frac{a^{2}}{h} \sqrt{\frac{\rho}{E}}(\omega[\mathrm{rad} / \mathrm{s}]$ is the circular frequency). Data: $E=73 . E 9[\mathrm{~Pa}], \nu=$ $0.34 \rho=2800\left[\mathrm{Kg} / \mathrm{m}^{3}\right] . \quad h=0.001[\mathrm{~m}]$; the three considered plate lengths are: $0.004,0.010,0.1[\mathrm{~m}]$

\begin{tabular}{cccc}
\hline $\mathrm{a} / \mathrm{h}$ & 4 & 10 & 100 \\
\hline $\mathrm{CLT}$ & 6.7318 & 7.0120 & 7.0688 \\
FSDT & 5.7765 & 6.7923 & 7.0664 \\
$N=1$ & 5.7764 & 6.7922 & 7.0664 \\
2 & 5.1397 & 5.8706 & 6.0572 \\
3 & 5.0704 & 5.8523 & 6.0570 \\
4 & 5.0688 & 5.8522 & 6.0570 \\
$\left(u_{z}=u_{z}^{0}\right) 4$ & 5.6314 & 6.7509 & 7.0660 \\
\hline
\end{tabular}

Table 2

One Layered orthotropic plate made of unidirectional lamina. Circular frequency parameter $\omega \times \frac{a^{2}}{h} \sqrt{\frac{\rho}{E_{T}}}(\omega[\mathrm{rad} / \mathrm{s}]$ is the circular frequency). Data: $E_{L}=50 . E 9$ (500.E9) [Pa], $E_{T}=10 . E 9 G_{L T}=G_{T T}=5 . E 9$ $\nu_{L T}=\nu_{T T}=0.25 \rho=1600\left[\mathrm{Kg} / \mathrm{m}^{3}\right], h=0.001[\mathrm{~m}]$; the three considered plate lengths are: $0.004,0.010,0.1[\mathrm{~m}])$

\begin{tabular}{cccccccc}
\hline & \multicolumn{3}{c}{$E_{L} / E_{T}=5$} & & \multicolumn{3}{c}{$E_{L} / E_{T}=50$} \\
\cline { 2 - 4 } \cline { 7 - 8 } $\mathrm{a} / \mathrm{h}$ & 4 & 10 & 100 & & 4 & 10 & 100 \\
\hline CLT & 6.7536 & 8.1324 & 8.5237 & & 15.668 & 20.748 & 20.916 \\
FSDT & 6.6926 & 8.1124 & 8.5199 & & 8.7161 & 15.538 & 20.832 \\
$N=1$ & 6.6948 & 8.1126 & 8.5199 & & 8.7160 & 15.538 & 20.832 \\
2 & 6.5853 & 7.9529 & 8.3422 & & 8.6810 & 15.490 & 20.766 \\
3 & 6.4207 & 7.8996 & 8.3416 & & 8.2853 & 14.913 & 20.750 \\
4 & 6.4193 & 7.8999 & 8.3416 & & 8.2833 & 14.912 & 20.749 \\
$\left(u_{z}=u_{z}^{0}\right) 4$ & 6.4993 & 8.0498 & 8.5192 & & 8.3058 & 14.950 & 20.815 \\
\hline
\end{tabular}

$F_{0}(z)=1, F_{1}(z)=z$.

Bold letter denotes arrays:

$$
u_{0}(x, y)=\left(u_{x_{0}}(x, y), u_{y_{0}}(x, y), u_{z_{0}}(x, y)\right), u_{1}(x, y)=\left(u_{x_{1}}(x, y), u_{y_{1}}(x, y), u_{z_{1}}(x, y)\right)
$$

The displacement unknowns are:

$$
u_{x_{0}}(x, y)=u_{x}^{0}, u_{y_{0}}=u_{y}^{0}, u_{z_{0}}(x, y)=u_{z}^{0}, u_{x_{1}}(x, y)=\phi_{x}, u_{y_{1}}=\phi_{y},
$$

and

$$
u_{z_{1}}(x, y,)=0
$$

This last condition underline that trasnverse normal strains are discarded in the FSDT formulated plate modelings.

Enhancement of FSDT to include transverse normal strain effects. Transverse normal strains can be included by removing the condition in Eq. (4). The explicit form of the displacement model is,

$$
\begin{aligned}
& u_{x}=F_{0}(z) u_{0_{x}}+F_{1}(z) u_{1_{x}} \\
& u_{y}=F_{0}(z) u_{0_{y}}+F_{1}(z) u_{1_{y}} \\
& u_{z}=F_{0}(z) u_{0_{z}}+F_{1}(z) u_{1_{z}}
\end{aligned}
$$

Higher order theories. The displacement model of previous paragraph can be easily extended to higher order Taylor expansion. Such an expansion can be herein written in array form,

$$
u(x, y, z)=F_{0}(z) u_{0}(x, y)+F_{1}(z) u_{1}(x, y)+\ldots+\ldots F_{N}(z) u_{N}(x, y)
$$

or, by introducing Einstein rule for repeated indexes,

$$
u(x, y, z)=F_{\tau}(z) u_{\tau}(x, y) \tau=0, N
$$




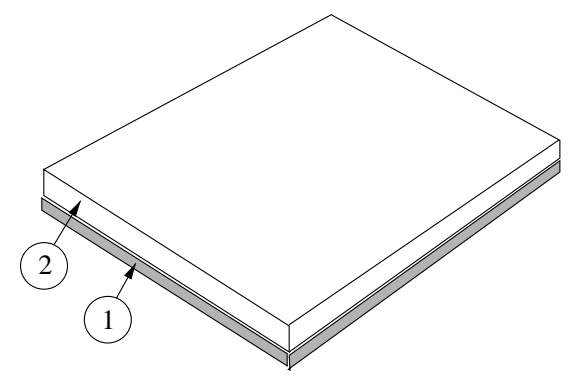

Fig. 4. Multilayered plates made of two different metallic materials.

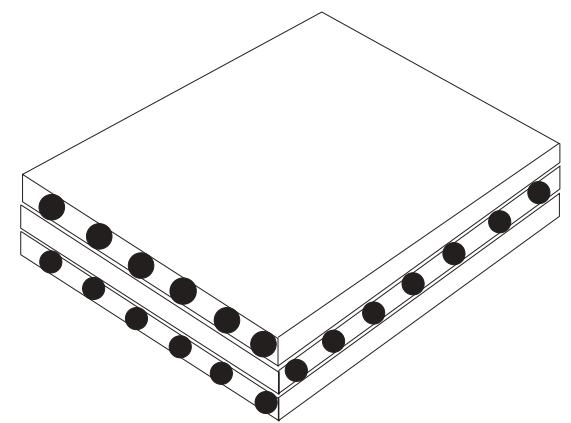

Fig. 5. Composite cross-ply plate.

where

$$
F_{\tau}(z)=z^{\tau}, \tau=0, N
$$

For convenience this displacement model is re-written according to the following notations,

$$
u(x, y, z)=F_{t}(z) u_{t}(x, y)+F_{b}(z) u_{b}(x, y)+F_{\tau}(z) u_{\tau}(x, y) \tau=2, N
$$

where

$$
F_{t}(z)=1, F_{b}(z)=z, F_{\tau}(z)=z^{\tau}, \tau=2, N
$$

With respect to Eq. (6), the constant and linear terms have been now denoted by subscript $t$ and $b$, respectively. Such a notation coincide to the one that will be used for the layer-wise cases.

Description of $\mathbf{Z Z}$ effect via Murakami's $\mathbf{Z Z}$ function. The considered displacement models are not able to describe ZZ effect. The discontinuity of the first derivative with correspondence to the layer interfaces, in the ESLM framework, can be introduced by employing the Murakami Zig-Zag Function (MZZF).

MZZF was proposed by Murakami [12] in the framework of Reissner Mixed Variational Theorem RMVT applications. The explicit form of MZZF is

$$
M(z)=(-1)^{k} \zeta_{k}
$$

The not dimensional layer coordinate $\zeta_{k}=z_{k} / 2 h_{k}$ is further introduced ( $h_{k}$ is the thickness of the $k$-th layer while $z_{k}$ is the layer thickness coordinate). $M(z)$ has the following properties:

- it is piece-wise linear function of the layer coordinates $z_{k}$;

- $M(z)$ has unit amplitude for the whole layers;

- the slope assumes opposite sign between two-adjacent layers (Its amplitude is layer thickness dependent).

The displacement including MZZF is written in the form,

$$
u=u_{0}+(-1)^{k} \zeta_{k} u_{Z}+z^{r} u_{r}, \quad r=1,2, . ., N
$$


Table 3

Two-layered plate made of metallic layers. $\omega \times \frac{a^{2}}{h} \sqrt{\frac{\rho^{1}}{E^{1}}}$ (subscript 1 denotes the first layer, starting from the plate bottom). Layers data: layer 1 coincide with the metallic plate of Table $1\left(h_{1}=0.001[\mathrm{~m}]\right)$; the second layer is made of steel $h_{2}=0.002, E_{2}=210 E 09[P a], \nu_{2}=0.3, \rho_{2}=$ $7800\left[\mathrm{~kg} / \mathrm{m}^{3}\right]$. The considered plate lengths are: $a=$ $0.012,0.030,0.300[\mathrm{~m}]$

\begin{tabular}{|c|c|c|c|}
\hline $\mathrm{a} / \mathrm{h}$ & 4 & 10 & 100 \\
\hline CLT & 5.8432 & 6.0347 & 6.0730 \\
\hline FSDT & 5.2087 & 5.8997 & 6.0726 \\
\hline$N=1$ & 5.2075 & 5.8968 & 6.0695 \\
\hline 2 & 4.6923 & 5.3222 & 5.3626 \\
\hline 3 & 4.6800 & 5.2266 & 5.3591 \\
\hline \multirow[t]{2}{*}{4} & 4.6790 & 5.2263 & 5.3589 \\
\hline & \multicolumn{3}{|c|}{$\mathrm{ZZ}$ included } \\
\hline 1 & 4.9199 & 5.5160 & 5.6616 \\
\hline 2 & 4.6900 & 5.2284 & 5.3586 \\
\hline \multirow[t]{2}{*}{3} & 4.6537 & 5.2196 & 5.3581 \\
\hline & \multicolumn{3}{|c|}{ IC included } \\
\hline 1 & 4.8403 & 5.7920 & 6.0535 \\
\hline 2 & 4.6552 & 5.2226 & 5.3616 \\
\hline 3 & 4.6666 & 5.2232 & 5.3587 \\
\hline \multirow[t]{2}{*}{4} & 4.6716 & 5.2243 & 5.3587 \\
\hline & \multicolumn{3}{|c|}{$\mathrm{ZZ}$ and IC included } \\
\hline 2 & 4.8073 & 5.4209 & 5.5723 \\
\hline 3 & 4.6687 & 5.2235 & 5.3585 \\
\hline \multirow[t]{2}{*}{4} & 4.6537 & 5.2196 & 5.3581 \\
\hline & \multicolumn{3}{|c|}{ Layer-Wise ZZ included } \\
\hline 1 & 4.9199 & 5.5160 & 5.6616 \\
\hline 2 & 4.6695 & 5.2230 & 5.3579 \\
\hline 3 & 4.6533 & 5.2193 & 5.3579 \\
\hline \multirow[t]{2}{*}{4} & 4.6532 & 5.2193 & 5.3579 \\
\hline & \multicolumn{3}{|c|}{ Layer-Wise ZZ and IC included } \\
\hline 1 & 4.7241 & 5.2847 & 5.4207 \\
\hline 2 & 4.6530 & 5.2193 & 5.3579 \\
\hline 3 & 4.6531 & 5.2193 & 5.3579 \\
\hline 4 & 4.6532 & 5.2193 & 5.3579 \\
\hline
\end{tabular}

Subscripts $Z$ refers to the introduced ZZ term. Higher order distributions in the $z$-direction are introduced by the $r$-polynomials.

In a unified form the displacement model is therefore rewritten

$$
u=F_{t} u_{t}+F_{b} u_{b}+F_{r} u_{r}=F_{\tau} u_{\tau}, \quad \tau=t, b, r, \quad r=1,2, . ., N
$$

Subscripts $b$ denotes values related to the plate reference surface $\Omega\left(u_{b}=u_{0}\right)$ while subscripts $t$ is now refers to the introduced zigzag term $\left(u_{t}=u_{Z}\right)$. The functions $F_{\tau}$ assume the following explicit form

$$
F_{b}=1, \quad F_{t}=M(z), \quad F_{r}=z^{r}, \quad r=1,2, . ., N
$$

Mixed theories that describe IC and ZZ Interlaminar continuous transverse shear and normal stress can be introduced by referring to RMVT applications [4]. According to RMVT an interlaminar continuous transverse stress field can be assumed in each layer:

$$
\sigma_{n M}^{k}=F_{t} \sigma_{n t}^{k}+F_{b} \sigma_{n b}^{k}+F_{r} \sigma_{n r}^{k}=F_{\tau} \sigma_{n \tau}^{k}, \quad \tau=t, b, r, \quad r=2,3, . ., N ; \quad k=1,2, . ., N_{l}
$$

This stress field can be used in along with with MZZF to obtain a theories that fulfill both IC and ZZ.

The meaning of the used polynomials will be given in the next paragraphs. 
Table 4

Multilayered cross ply plate $0 / 90 / 0$. Circular frequency parameter $\bar{\omega}=\omega \sqrt{a^{4} \rho / E_{T} h^{2}}$. Data of the layers in non dimensioned form: $\frac{E_{L}}{E_{T}}=40, \frac{G_{L T}}{E_{T}}=0.5, \frac{G_{T T}}{E_{T}}=0.60 ; \nu_{L T}=v_{T T}=0.25 . \rho=1$. $h_{1} / h=h_{3} / h=0.25, h_{2} / h=0.50$

\begin{tabular}{|c|c|c|c|c|c|}
\hline $\mathrm{a} / \mathrm{h}$ & 2 & 4 & 10 & 20 & 100 \\
\hline CLT & 15.830 & 17.907 & 18.652 & 18.767 & 18.804 \\
\hline FSDT & 5.492 & 9.379 & 10.820 & 17.583 & 18.751 \\
\hline \multicolumn{6}{|c|}{ No ZZ No IC } \\
\hline$N=1$ & 5.927 & 9.960 & 15.573 & 17.829 & 18.833 \\
\hline 2 & 5.927 & 9.960 & 15.573 & 17.829 & 18.833 \\
\hline 3 & 5.393 & 9.394 & 15.271 & 17.717 & 18.827 \\
\hline 4 & 5.393 & 9.394 & 15.271 & 17.717 & 18.827 \\
\hline \multicolumn{6}{|c|}{$\mathrm{ZZ}$ included } \\
\hline 1 & 5.926 & 9.956 & 15.563 & 17.817 & 18.819 \\
\hline 2 & 5.920 & 9.938 & 15.522 & 17.763 & 18.760 \\
\hline 3 & 5.390 & 9.388 & 15.232 & 17.655 & 18.754 \\
\hline \multicolumn{6}{|c|}{ IC included } \\
\hline 1 & 5.572 & 9.522 & 15.260 & 17.703 & 18.826 \\
\hline 2 & 5.781 & 9.757 & 15.387 & 17.709 & 18.757 \\
\hline 3 & 5.365 & 9.364 & 15.221 & 17.652 & 18.754 \\
\hline 4 & 5.361 & 9.367 & 15.224 & 17.653 & 18.754 \\
\hline \multicolumn{6}{|c|}{$\mathrm{ZZ}$ an IC included } \\
\hline 1 & 5.782 & 9.768 & 15.423 & 17.759 & 18.814 \\
\hline 2 & 5.847 & 9.846 & 15.455 & 17.737 & 18.758 \\
\hline 3 & 5.370 & 9.371 & 15.224 & 17.652 & 18.754 \\
\hline \multicolumn{6}{|c|}{ Layer-Wise (D) No IC } \\
\hline 1 & 5.414 & 9.473 & 15.335 & 17.703 & 18.761 \\
\hline 2 & 5.277 & 9.236 & 15.152 & 17.626 & 18.753 \\
\hline 3 & 5.262 & 9.224 & 15.148 & 17.626 & 18.753 \\
\hline 4 & 5.260 & 9.224 & 15.148 & 17.626 & 18.753 \\
\hline \multicolumn{6}{|c|}{ Layer-Wise (M) ZZ and IC } \\
\hline 1 & 5.143 & 9.103 & 15.087 & 17.604 & 18.752 \\
\hline 2 & 5.247 & 9.220 & 15.148 & 17.626 & 18.753 \\
\hline 3 & 5.259 & 9.224 & 15.148 & 17.626 & 18.753 \\
\hline 4 & 5.260 & 9.224 & 15.148 & 17.626 & 18.753 \\
\hline
\end{tabular}

Layer Wise theories Layer-wise description requires assuming independent displacement variables in each $k$-layer. The thickness expansion used for ESLM cases Eq. (11) is not convenient for layer-wise description. Interlaminar continuity for displacements can be more conveniently imposed by employing interface values as unknown variables. Therefore, layer-wise description is written according to the following expansion,

$$
u_{n M}^{k}=F_{t} u_{n t}^{k}+F_{b} u_{n b}^{k}+F_{r} u_{n r}^{k}=F_{\tau} u_{n \tau}^{k}, \quad \tau=t, b, r, \quad r=2,3, . ., N ; \quad k=1,2, . ., N_{l}
$$

In contrast to what in Eq. (11), it is now intended that the subscripts $t$ and $b$ denote values related to the layer top and bottom surface, respectively. In fact, they consist of the linear part of the expansion.

The thickness functions $F_{\tau}\left(\zeta_{k}\right)$ have been defined by

$$
F_{t}=\frac{P_{0}+P_{1}}{2}, \quad F_{b}=\frac{P_{0}-P_{1}}{2}, \quad F_{r}=P_{r}-P_{r-2}, \quad r=2,3, . ., N
$$

in which $P_{j}=P_{j}\left(\zeta_{k}\right)$ is the Legendre polynomial of the $j$-order defined in the $\zeta_{k}$-domain: $-1 \leqslant \zeta_{k} \leqslant 1$. Fourth order case will be used in the numerical investigations; related polynomials are

$$
P_{0}=1, \quad P_{1}=\zeta_{k}, \quad P_{2}=\left(3 \zeta_{k}^{2}-1\right) / 2, \quad P_{3}=\frac{5 \zeta_{k}^{3}}{2}-\frac{3 \zeta_{k}}{2}, \quad P_{4}=\frac{35 \zeta_{k}^{4}}{8}-\frac{15 \zeta_{k}^{2}}{4}+\frac{3}{8}
$$

The chosen functions have the following properties:

$$
\zeta_{k}=\left\{\begin{aligned}
1: F_{t} & =1 ; F_{b}=0 ; F_{r}=0 \\
-1: F_{t} & =0 ; F_{b}=1 ; F_{r}=0
\end{aligned}\right.
$$


Table 5

\begin{tabular}{|c|c|c|c|c|c|c|}
\hline & \multicolumn{3}{|c|}{$a / h=4$} & \multicolumn{3}{|c|}{$a / h=50$} \\
\hline & First & Second & Third & First & Second & Third \\
\hline Exact [9] & 57074.5 & 191301 & 250769 & 618.118 & 15681.6 & 21492.8 \\
\hline FSDT & 73975.0 & 198465 & 286786 & 689.817 & 15877.2 & 22943.9 \\
\hline \multirow[t]{2}{*}{ CLT } & 198465 & 286796 & - & 692.218 & 15877.2 & 22943.2 \\
\hline & \multicolumn{6}{|c|}{$\mathrm{ZZ}$ and IC discarded } \\
\hline$N=1$ & 73942.1 & 196015 & 266284 & 689.204 & 15695.0 & 21507.4 \\
\hline 2 & 68240.0 & 195828 & 261780 & 605.266 & 15694.9 & 21505.0 \\
\hline 3 & 58073.6 & 195785 & 259064 & 603.451 & 15694.2 & 21499.8 \\
\hline \multirow[t]{2}{*}{4} & 57939.5 & 194524 & 254384 & 603.229 & 15693.5 & 21497.6 \\
\hline & \multicolumn{6}{|c|}{ ZZ included IC discarded } \\
\hline 1 & 63152.5 & 195956 & 266092 & 687.266 & 15693.6 & 21498.4 \\
\hline 2 & 59935.1 & 195677 & 260335 & 603.926 & 15693.5 & 21496.3 \\
\hline \multirow[t]{2}{*}{3} & 56996.1 & 195667 & 259022 & 603.267 & 15687.0 & 21496.3 \\
\hline & \multicolumn{6}{|c|}{ Layer-wise ZZ included and IC discarded } \\
\hline 1 & 57252.5 & 194840 & 255646 & 619.022 & 15683.4 & 21499.4 \\
\hline 2 & 57081.9 & 191311 & 250786 & 618.105 & 15681.6 & 21492.6 \\
\hline 3 & 57074.0 & 191301 & 250768 & 618.104 & 15681.6 & 21492.6 \\
\hline 4 & 57074.0 & 191301 & 250768 & 618.104 & 15681.6 & 21492.6 \\
\hline
\end{tabular}

Table 6

Elastic, piezoelectric and dielectric properties of used materials

\begin{tabular}{lcc}
\hline Property & PZT-4 & Gr/Ep \\
\hline$E_{1}[\mathrm{GPa}]$ & 81.3 & 132.38 \\
$E_{2}[\mathrm{GPa}]$ & 81.3 & 10.756 \\
$E_{3}[\mathrm{GPa}]$ & 64.5 & 10.756 \\
$\nu_{12}[-]$ & 0.329 & 0.24 \\
$\nu_{13}[-]$ & 0.432 & 0.24 \\
$\nu_{23}[-]$ & 0.432 & 0.49 \\
$G_{44}[\mathrm{GPa}]$ & 25.6 & 3.606 \\
$G_{55}[\mathrm{GPa}]$ & 25.6 & 5.6537 \\
$G_{66}[\mathrm{GPa}]$ & 30.6 & 5.6537 \\
$e_{15}\left[\mathrm{C} / \mathrm{m}^{2}\right]$ & 12.72 & 0 \\
$e_{24}\left[\mathrm{C} / \mathrm{m}^{2}\right]$ & 12.72 & 0 \\
$e_{31}\left[\mathrm{C} / \mathrm{m}^{2}\right]$ & -5.20 & 0 \\
$e_{32}\left[\mathrm{C} / \mathrm{m}^{2}\right]$ & -5.20 & 0 \\
$e_{33}\left[\mathrm{C} / \mathrm{m}^{2}\right]$ & 15.08 & 0 \\
$\tilde{\epsilon}_{11} / \epsilon_{0}[-]$ & 1475 & 3.5 \\
$\tilde{\epsilon}_{22} / \epsilon_{0}[-]$ & 1475 & 3.0 \\
$\tilde{\epsilon}_{33} / \epsilon_{0}[-]$ & 1300 & 3.0 \\
\hline
\end{tabular}

Mixed Layer-Wise theories that describe IC. The layer-wise description used for displacements is in this case expended to transverse stresses:

$$
\begin{array}{lr}
u^{k}=F_{t} u_{t}^{k}+F_{b} u_{b}^{k}+F_{r} u_{r}^{k}=F_{\tau} u_{\tau}^{k} & \tau=t, b, r \\
\sigma_{n M}^{k}=F_{t} \sigma_{n t}^{k}+F_{b} \sigma_{n b}^{k}+F_{r} \sigma_{n r}^{k}=F_{\tau} \sigma_{n \tau}^{k} & k=2,3, . ., N \\
& =1,2, . ., N_{l}
\end{array}
$$

The top and bottom values have also been used as unknown variables. The interlaminar transverse shear and normal stress continuity can be therefore easily linked:

$$
\sigma_{n t}^{k}=\sigma_{n b}^{(k+1)}, k=1, N_{l}-1
$$

\section{Results}

In order to assess the discussed theories, a number of plate problems were considered. Results on free vibration are given in the following paragraphs. 


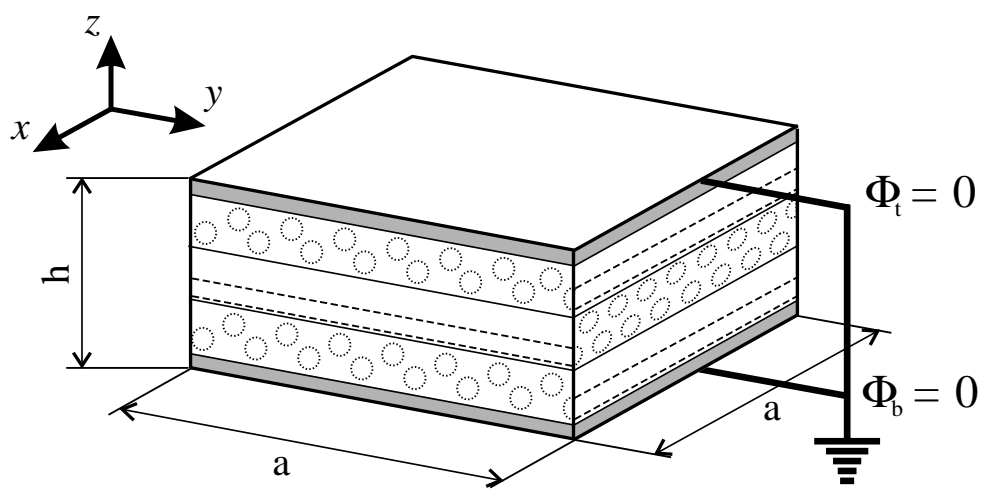

Fig. 6. Piezoelectric plates.

These all refer to simply supported, square plates for which a closed form solution of the Navier type can be obtained.

Plate made of one layer. First a homogeneous isotropic plate was investigated. The geometrical parameters are given in Fig. 2.

The results are quoted in Table 1 . Thick $(a / h=4)$, moderately thick $(a / h=10)$ and thin $(a / h=100)$ plate results are compared. The CLT and FSDT results are compared to higher order theories that encompass linear to fourth order expansions in the thickness coordinate $z$.

The following comments can be made:

- CLT analysis is confirmed to be adequate for homogeneous thin plates, and moderately thick plates with respect to FSDT results, that is the effect of transverse shear deformation can be neglected in thin plates.

- Higher order terms can improve FSDT solutions in both thick and thin geometries. It was found that such an improvement is mainly due to the retainment of transverse normal strains (as shown by the results in the last row).

- Higher order expansions $(N=3,4)$ for both in-plane and out-of-plane displacements are required to obtain an accurate free vibrational response for both thick and thin plates.

An orthotropic plate made of unidirectional lamina of a composite material is considered in Fig. 3 and Table 2. Two values of the orthotropic ratio $E_{L} / E_{T}$ ( $L$ and $T$ denote the Longitudinal and Transverse fiber directions, respectively, see Fig. 3 and the book by Reddy [15]) were investigated: low modulus $E_{L} / E_{T}=5$ and high modulus composite $E_{L} / E_{T}=50$. In addition to the comments that have already been made, one can notice that the effect of transverse shear deformation increases as the orthotropic ratio increases. As a consequence, CLT can lead to large errors even though moderately thick plates are being analyzed. The transverse strain effects are less important with respect to the Table 1 results. However, a more detailed description of the transverse normal strain effects on laminated structures can be found in Carrera [3]).

The Two layered plate made of two homogeneous materials. This type of plate, made of two different metallic materials (Fig. 4), is very common in thermal protected structures. The discontinuity of the mechanical properties at the interface between the two layers permits one to analyze the role played by ZZ and IC effects as well as to compare Equivalent Single Layers analyses with Layer-Wise ones. The results and data are given in Table 3. Analyses that have already been considered are compared to advanced Zig-Zag theories. According to a previous work [6], the LWM related to the forth order $(N=4)$ expansion should be considered as the exact solution. As the multilayered plate is an unsymmetrically laminate, the benefits of even values of $N$ are more evident with respect to the results of Tables 1 and 2. Higher order classical theories that discard IC and ZZ are not able to capture the exact circular frequency parameter in thick plate cases. Zig-Zag theories are required for this aim. Lower order expansions are usually required by layer-wise analyses with respect to ESLM ones. 
The Multilayered plate made of composite materials. The three-layered symmetrically laminated cross/ply plate of Fig. 5 was investigated as shown in Table 4 . Very thick $(a / h=2)$ plate case was included in the comparison. Even though this is a not realistic plate, such a geometry permits one to explore the performance of a given refined plate theory to capture three-dimensional effects. It can be concluded that layer-wise analyses are required for these geometries.

The piezo-electric plate. A coupled electromechanical problem was considered as shown in Fig. 6 and Table 5. It consisted of five layers which were assumed to be perfectly bonded to each other. The top and bottom layers are made of piezoelectric materials with the thickness $h_{p}^{k}=0.1 \mathrm{~h}$ each, the three structural layers of equal thickness had the configuration 0/90/0. Heyliger and Saravanos [9] provided an exact solution to this problem. The materials were PZT-4 (which is a Piezoelectric Lead Zirconate ceramics materials) for the piezoelectric layers and Gr/Ep (which is a graphite epoxy composite materials) for the structural layers. The corresponding material properties can be taken from Table 6 . Two thickness ratios $a / h=4$ and 50 were considered. Electrical potential $\Phi$ has been described as the transverse stresses. The electrical stiffness has been fully taken into account. The conclusions that had previously been made were confirmed for piezoelectric plates. First, second and third circular frequencies related to the first three fundamental modes were compared. It can be observed that the accuracy of the various theories is very much subordinate to the considered mode. Such a conclusion, even though not documented in the previous tables, could be confirmed for all the plate considered in the previous examples.

\section{Concluding remark}

The paper has presented an assessment of the classical and zig-zag theories to evaluate the free vibration response of multilayered problems. Attention was restricted to closed form solutions of simply supported plates composed of orthotropic layers. Homogeneous, composite and piezo-electric layers were considered. More than twenty theories were compared for the same cases. The conducted assessment could serve as a desk-bed for specialists in sound, shock and vibration to help them to choose the most appropriate theories for a given plate problem.

\section{References}

[1] S.A. Ambartsumian, On a theory of bending of anisotropic plates, Investiia Akad. Nauk SSSR, Ot. Tekh. Nauk. (4) (1958).

[2] A.E. Bogdonovic and R. Sierakowsky, Composite Materials and Structures: Science Technology and Application 52, 551-366.

[3] E. Carrera, A Study of Transverse Normal Stress Effects on Vibration of Multilayered Plates and Shells 225 (1999), 803-829.

[4] E. Carrera, Developments, Ideas and Evaluations based upon the Reissner's Mixed Theorem in the Modeling of Multilayered Plates and Shells 54 (2001), 301-329.

[5] E. Carrera, Theories and Finite Elements for Multilayered Anisotropic, Composite Plates and Shells 9 (2002), 87-140.

[6] E. Carrera, Historical review of Zig-Zag theories for multilayered plates and shells 56 (2003), 287-308.

[7] E. Carrera, Theories and Finite Elements for Multilayered Plates and Shells: A Unified Compact Formulation with Numerical Assessment and Benchmarking 10 (2003), 215-297.

[8] E.I. Grigolyuk and G.M. Kulikov, General Directions of the Development of Theory of Shells 24 (1988), $287-298$.

[9] P. Heyliger and D.A. Saravanos, Exact free-vibration analysis of laminated plates with embedded piezoelectric layers, Journal of the Acoustical Society of America 98 (1995), 1547-1557.

[10] S.G. Lekhnitskii, Strength Calculation of Composite Beams, Vestnik inzhen. i tekhnikov (9) (1995).

[11] S.A. Lur'e and N.P. Shumova, Kinematic models of refined theories concerning composite beams, plates and shells 32 (1996), $422-430$.

[12] H. Murakami, Laminated composite plate theory with improved in-plane responses 53 (1986), 661-666.

[13] M.S. Qatu, Recent research advances in the dynamic behavior of shells: 1989-2000, Part 1: Laminated composite shells 55 (2002), 325-350.

[14] M.S. Qatu, Recent research advances in the dynamic behavior of shells: 1989-2000, Part 2: Homogeneous shells 55 (2002), $415-434$.

[15] J.N. Reddy, Mechanics of Laminated Composite Plates, Theory and Analysis. CRC Press, 1997.

[16] J.N. Reddy and D.H. Robbins, Theories and computational models for composite laminates 47 (1994), $147-165$.

[17] E. Reissner, On a certain mixed variational theory and a proposed application 20 (1984), 1366-1368.

[18] V.V. Vasilive and S.A. Lur'e, On refined theories of beams, plates and shells 26 (1992), 422-430. 

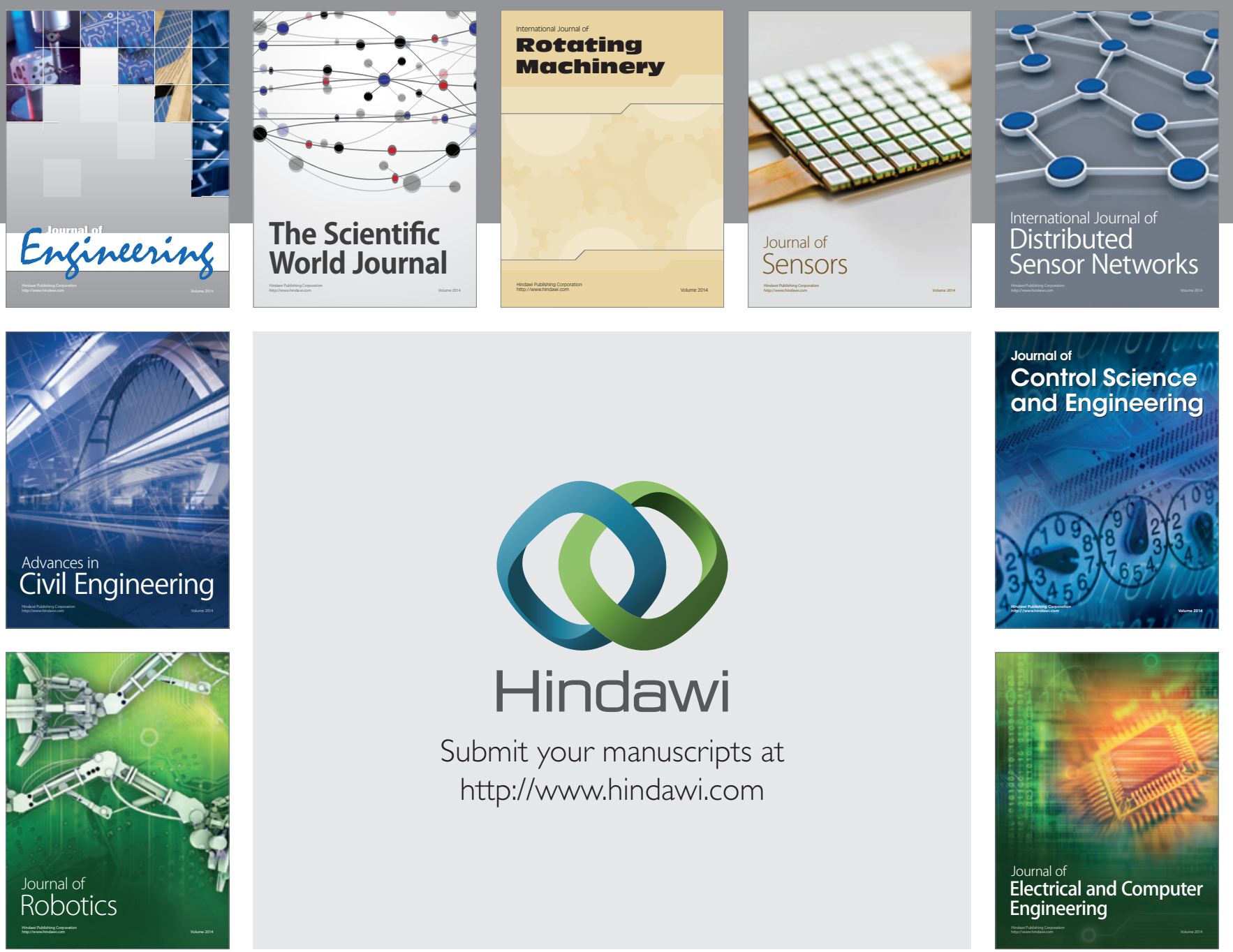

Submit your manuscripts at

http://www.hindawi.com
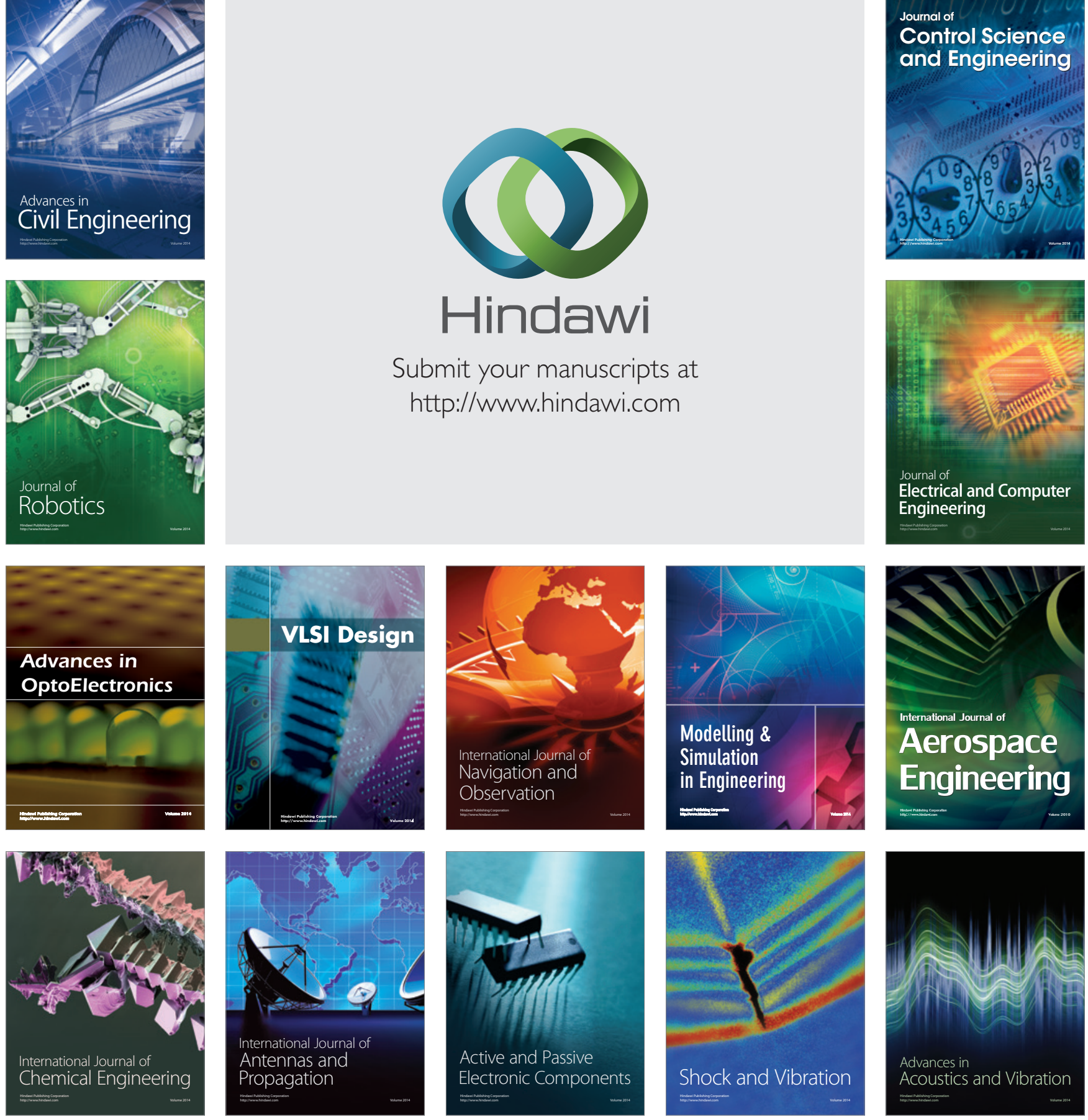